\title{
Burden and Factors Associated with Schistosomiasis and Soil-Transmitted Helminth Infections Among School-Age Children in Huambo, Uige and Zaire provinces, Angola
}

Adam W. Bartlett ( $\square$ abartlett@kirby.unsw.edu.au )

University of New South Wales https://orcid.org/0000-0002-7532-9316

Jose C. Sousa-Figueiredo

Health Research Center Angola

Roelofje C. van Goor

The Mentor Initiative

Paul Monaghan

The Mentor Initiative

Warren Lancaster

The END Fund

Rukaaka Mugizi

The Mentor Initiative

Elsa P. Mendes

Ministry of Health of Angola

Susana Vaz Nery

University of New South Wales

Sergio Lopes

The Mentor Initiative https://orcid.org/0000-0002-8973-948X

\section{Research Article}

Keywords:

Posted Date: February 3rd, 2022

DOI: https://doi.org/10.21203/rs.3.rs-1302136/v1

License: (c) (1) This work is licensed under a Creative Commons Attribution 4.0 International License. Read Full License 


\section{Abstract}

Background: Schistosomiasis and soil-transmitted helminths (STHs) contribute high disease burdens amongst the neglected tropical diseases (NTDs) and are public health problems in Angola. This study reports the prevalence, intensity and risk factors for schistosomiasis and STH infection in Huambo, Uige and Zaire provinces, Angola, to inform a school-based preventive chemotherapy program.

Methods: A two-stage cluster design was used to select schools and schoolchildren to participate in parasitological and water, sanitation and hygiene (WASH) surveys across Huambo, Uige, and Zaire provinces. Point-of-care circulating cathodic antigen and urinalysis rapid diagnostic tests (RDTs) were used to determine the prevalence of Schistosoma mansoni and S. haematobium, respectively. Kato-Katz was used to identify and quantify STH species and quantify and compare with RDTs for $S$. mansoni. Urine filtration was used to quantify and compare with RDTs for S. haematobium. Descriptive statistics were used for prevalence and infection intensity of schistosomiasis and STH infection. Performance of RDTs was assessed through specificity and Cohen's Kappa agreement with microscopy. A multivariate regression analysis was used to determine demographic and WASH factors associated with schistosomiasis and STH infection.

Results: A total 575 schools and 17,093 schoolchildren participated in the schistosomiasis survey, of which 121 schools and 3,649 schoolchildren participated in the STH survey. Overall prevalence of S. mansoni was 21.2\% (municipality range $0.9-74.8 \%$ ) and S. haematobium $13.6 \%$ (range $0-31.2 \%$ ), with an overall prevalence of schistosomiasis of $31.4 \%$ (range $5.9-77.3 \%$ ). Overall prevalence of $A$. lumbricoides was $25.1 \%$ (range $0-89.7 \%$ ), hookworm $5.2 \%$ (range $0-42.6 \%$ ), and T. trichiura 3.6\% (range 0-24.2\%), with an overall prevalence of STH infection of 29.5\% (0.8-89.7\%). Ecological zone and ethnicity were factors associated with schistosomiasis and STH infection, with older age and female sex additional risk factors for S. haematobium.

Conclusions: Most municipalities met WHO-defined prevalence thresholds for a schistosomiasis preventive chemotherapy program. A STH preventive chemotherapy program is indicated for nearly all municipalities in Uige and select municipalities in Huambo and Zaire. The association between ecological zone and ethnicity with schistosomiasis and STH infection necessitates further evaluation of home and school environmental, sociodemographic and behavioural factors to inform targeted control strategies to complement preventive chemotherapy programs.

\section{Background}

Schistosomiasis (infection caused by Schistosoma species) and soil-transmitted helminth (STH) infections including hookworms (Necator americanus, Ancylostoma duodenale, A. ceylanicum), Ascaris lumbricoides, and Trichuris trichiura are prevalent in tropical and subtropical areas, particularly in lowresourced communities with limited access to good quality water sources, sanitation and hygiene [1]. They cause a range of gastrointestinal and urogenital disease, that can lead to anaemia and malnutrition, and occasionally death [1]. School-age children are particularly susceptible to these infections as they are frequently exposed to contaminated soil and water (through playing, washing, eating and drinking) with less awareness of sanitation and hygiene, during an important period of physical and cognitive development [1]. STH infections contribute the greatest burden of disease of the neglected tropical diseases (NTDs), accounting for over 3.3 million disease-adjusted life years (DALYs) [2]. Schistosomiasis accounts for over 1.8 million DALYs, the third highest burden attributable to a NTD behind STH infections and dengue [2]. As such, the World Health Organization (WHO) have targeted schistosomiasis and STH infections for elimination as a public health problem by 2030 [3]. For schistosomiasis, this means reducing the proportion of heavy intensity infection to $<1 \%$ in school-age children; while for STH infection, this means decreasing the proportion of moderate and heavy intensity infection to $<2 \%$ in school-age children [3-5].

An integral component to schistosomiasis and STH control programs is the implementation of large-scale preventive chemotherapy programs, consisting of regular distribution of anthelminthic therapy to entire populations or targeted to at-risk populations such as school-age children [1]. The WHO recommends the prevalence of such infections as the main indicator for preventive chemotherapy programs and the frequency at which anthelminthic therapy is delivered $[1,6]$. In addition to preventive chemotherapy, strategies to increase access to clean water, improved sanitation and adequate hygiene practices are recommended for sustainable control of schistosomiasis and STH infections [3].

Diagnostic techniques that facilitate efficient and cost-effective field work increase the capacity of field teams to survey larger cohorts with greater geographic spread, thereby sampling a more representative sample of the population being assessed for control measures. There has been evolving interest in the use of rapid diagnostic tests (RDTs) for schistosomiasis, with the first large-scale prevalence survey using schistosomiasis RDTs undertaken in Namibia in 2012 and 2013 [7]. This prevalence survey, using schistosomiasis RDTs to detect $S$. mansoni and S. haematobium, was able to survey 17,896 schoolchildren from 299 schools, and demonstrated a sensitivity above $80 \%$ and specificity above 95\% for both RDTs when compared to the WHO reference microscopy techniques (Kato-Katz and urine filtration). Additional large-scale schistosomiasis RDT surveys have since been conducted that build upon the field work capacity and role of schistosomiasis RDTs in informing the implementation and impact of schistosomiasis control programs [8-12].

Schistosomiasis and STH infections have long been recognised as public health problems in Angola. A cross-sectional survey in 2010 across three communes in Bengo province demonstrated the prevalence of at least one STH infection as $22.6 \%$ in preschool children and $31.6 \%$ in school-age children; and the prevalence of microhaematuria (as a proxy for $S$. haematobium) as $10.0 \%$ and $16.6 \%$ in preschool and school-age children respectively [13]. A crosssectional survey (2013-2014) in children in Cubal estimated a crude prevalence of urinary schistosomiasis (by the presence of haematuria) of 61.2\% [14]. Recognising the burden of schistosomiasis and STH infection, the Ministry of Health in Angola undertook a school-based preventive chemotherapy program with albendazole in 2013 followed by a school-based preventive chemotherapy program with praziquantel and albendazole from 2014. To inform the implementation of the program from 2014 onwards in each province, parasitological and water, sanitation and hygiene (WASH) surveys were carried out in stages across the country, with Huambo, Uige and Zaire the first three provinces to be surveyed. This analysis reports the results from those surveys 
including: (i) the prevalence and intensity of schistosomiasis and STH infections; (ii) comparing the performance of RDTs and microscopy in diagnosing schistosomiasis; and (iii) demographic and WASH factors associated with schistosomiasis and STH infection.

\section{Methods}

\section{Study design and sampling}

A two-stage cluster design was employed to select schools and schoolchildren to participate in the surveys. The surveys consisted of: (i) a RDT survey to determine the prevalence of S. mansoni and S. haematobium; and (ii) a microscopy survey to detect and quantify STH infection (hookworm, $A$. Iumbricoides, and $T$. trichiura), as well as quantify and provide diagnostic comparison with RDTs for $S$. mansoni and $S$. haematobium. The primary sampling frame was all registered primary and combined schools across Huambo, Uige and Zaire provinces. Following a similar approach to the schistosomiasis and STH mapping conducted in Namibia [7], sample size calculations for schistosomiasis prevalence were conducted using an estimated prevalence of 25\%, precision of 5\%, confidence level of $95 \%$ and design effect of 2.5 (using Epi Info v7.1.2; CDC Atlanta, USA). Based on these sample size calculations and the number of registered schools across Huambo, Uige and Zaire, a mapping resolution of 1 in 4 schools was deemed sufficient, resulting in 575 schools to be surveyed across the three provinces. The one in four schools for the schistosomiasis survey were selected using systematic random sampling and was adjusted to ensure representation from each municipality in each province. Of schools selected for the schistosomiasis survey, one in five were selected to participate in the STH survey. This sampling strategy resulted in 121 schools to be surveyed for STHs, with representation from each municipality, which provides a larger sample size than the WHO recommended five to ten schools for each of the three ecological zones in Angola.[1] In selecting schoolchildren, a sampling frame was created of all children attending school on the day of the survey, from which 30 children (15 males and 15 females) were selected using systematic random sampling.

\section{Field work}

Field teams collected data per province in three periods in 2014. Zaire province data collection took place between 23rd March and 2nd April, Uige province data collection occurred between 2nd June and 18th July, and Huambo province data collection was undertaken between 23rd June and 12th August.

\section{Survey procedures}

Field work teams were designated to either perform schistosomiasis RDTs or perform microscopy to detect and quantify STH and Schistosoma species. These teams operated independently in the field but were coordinated for schools selected to participate in both the schistosomiasis and STH surveys. On the day of the survey, selected schools were visited by field teams to explain the surveys to the school director and obtain written consent for the school and the schoolchildren to participate. Schoolchildren were selected using systematic random sampling of children present at the school on the day of the parasitological survey. Each participating child was given a single sample collection vial for urine, and those participating in the STH survey were also provided with a single sample collection vial for stool. The samples were returned to the field workers and analysed on the same day. Upon receipt of samples, the age of each child was recorded, and all participating children were provided with one tablet of albendazole and the appropriate number of tablets of praziquantel, as determined using a dose pole. For the schistosomiasis survey, technicians used the point-of-care circulating cathodic antigen (POC-CCA $\AA$ ) test to detect S. mansoni and Hemastix ${ }^{\circledR}$ to detect haematuria as a proxy for $S$. haematobium. For the STH survey, microscopists used the Kato-Katz technique (single smear) to detect and quantify STH species (hookworm, A. lumbrocoides and T. trichiura). Field teams performing microscopy also analysed specimens to detect and quantify S. mansoni and S. haematobium using Kato-Katz and the urine filtration technique respectively. A questionnaire was conducted in an interview with the school director of each school to evaluate: (i) the presence and functionality of latrines at the school; (ii) the accessibility to safe drinking water at the school; (iii) whether the schoolchildren had previously received preventive chemotherapy for schistosomiasis and STHs; and (iv) their knowledge of schistosomiasis. Data was entered manually into data entry forms by the field workers. The data entry forms were collated then the data was manually entered into an electronic database.

\section{Detection and classification of infection}

Single urine samples were collected from all schoolchildren to evaluate the prevalence of $S$. mansoni and S. haematobium using RDTs. The POC-CCA ${ }^{\circledR}$ results were graded as "negative", "trace", "+", "++" and "+++". The Hemastix® results were graded as "negative", "trace not haemolysed", "trace haemolysed", "+", "++" and "+++". Single stool samples were used for microscopy with the Kato-Katz technique to detect and determine the number of eggs per gram of faeces for STH species and S. mansoni [1]. The urine filtration technique was used to determine the number of $S$. haematobium eggs per 10mL of urine [1].

\section{Ethics statement}

Approval for the survey protocol was obtained by the Ministry of Public Health of Angola (101/GD/DNSP/2014). Informed written consent was obtained from the school directors of each school to permit participation of children in the school-based surveys.

\section{Statistical analysis}

Descriptive statistics were used to report the characteristics of the schools and schoolchildren that participated in the schistosomiasis and STH surveys for each municipality across Huambo, Uige and Zaire provinces. Prevalence, with 95\% confidence intervals (Cls), for schistosomiasis and STH infection was calculated for each municipality with adjustments made for the cluster survey design. Prevalence was separately determined for when RDT trace readings were considered positive and for when they were considered negative. Descriptive statistics were used to report infection intensity for STH and Schistosoma species as determined by microscopy. The spatial distribution of schistosomiasis and STH prevalence was represented graphically using QGIS version 3.18 . Diagnostic agreement between the RDTs (for trace readings as positive and trace readings as negative) and microscopy was performed using Cohen's 
Kappa agreement statistics (very good, $\mathrm{k}>0.8$; good, $0.6<\mathrm{K} \leq 0.8$; moderate, $0.4<\mathrm{K} \leq 0.6$; fair, $0.2<\mathrm{K} \leq 0.4$; poor, $\mathrm{K} \leq 0.2$ ). The specificity of the RDTs in detecting $S$. mansoni and $S$. haematobium was calculated using Kato-Katz and urine filtration as the comparator, respectively. A logistic regression analysis was used, using a mixed-effects model and adjusted for the cluster survey design, to assess factors associated with specific Schistosoma or STH species, as well as any schistosomiasis or STH infection. Covariates with a p-value $<0.2$ on univariate logistic regression analysis were included in a multivariate logistic regression analysis. The multivariate logistic regression analysis was conducted to determine adjusted odds ratios (aORs) in a stepwise fashion maintaining covariates that retained a p-value $<0.05$. Covariates included sex, age, school setting (rural, urban), ecological zone (Northern-coastal, Coffee, Central highland), ethnicity (Kikongo, Kimbundo, Umbundo), previous school deworming in 2013, presence of functional latrines at school, and presence of a reliable safe drinking water source at school. The primary logistic regression analysis was conducted considering infection determined by RDT trace readings as positive, with a secondary logistic regression analysis performed considering RDT trace readings as negative. All statistical analyses were performed using Stata version 17.0 (StataCorp LP, College Station, Texas).

\section{Results}

\section{Survey population}

There were a total 575 schools across Huambo, Uige and Zaire provinces from which 17,093 schoolchildren participated in the schistosomiasis survey. From this, 121 schools and 3,649 schoolchildren also participated in the STH survey. Overall, there was equal representation of males and females, and the median age of participants was 11 (interquartile range (IQR) 10,13) years. Table 1 shows the demographics of the schistosomiasis and STH survey populations for each province, and Additional File 1 provides the demographics of the schistosomiasis and STH surveys for each municipality.

Table 1

Demographics of participants in the schistosomiasis and soil-transmitted helminth surveys for Huambo, Uige and Zaire provinces.

\begin{tabular}{|c|c|c|c|c|c|c|c|c|}
\hline & \multicolumn{2}{|l|}{ Huambo } & \multicolumn{2}{|l|}{ Uige } & \multicolumn{2}{|l|}{ Zaire } & \multicolumn{2}{|l|}{ Total } \\
\hline & RDT & Microscopy & RDT & Microscopy & RDT & Microscopy & RDT & Microscopy \\
\hline & $N=7620$ & $N=1501$ & $\mathrm{~N}=7793$ & $\mathrm{~N}=1818$ & $\mathrm{~N}=1680$ & $\mathrm{~N}=330$ & $\mathrm{~N}=17093$ & $N=3649$ \\
\hline Schools & 254 & 50 & 265 & 60 & 56 & 11 & 575 & 121 \\
\hline \multicolumn{9}{|l|}{ Students } \\
\hline Male & 3796 (49.2\%) & 750 (50.0\%) & $3916(50.3 \%)$ & 903 (49.7\%) & $838(49.9 \%)$ & $165(50.0 \%)$ & $8550(50.0 \%)$ & $1818(49.8 \%)$ \\
\hline Female & 3824 (50.2\%) & $751(50.0 \%)$ & 3877 (49.8\%) & $915(50.3 \%)$ & $842(50.1 \%)$ & $165(50.0 \%)$ & $8543(50.0 \%)$ & $1831(50.2 \%)$ \\
\hline Age, years (IQR) & $11(10,13)$ & $11(10,13)$ & $11(10,13)$ & $11(9,13)$ & $12(10,13)$ & $12(10,14)$ & $11(10,13)$ & $11(10,13)$ \\
\hline \multicolumn{9}{|l|}{ Setting } \\
\hline Rural & $6150(80.7 \%)$ & $1411(94.0 \%)$ & $6740(86.6 \%)$ & $1643(90.5 \%)$ & $1350(80.4 \%)$ & $270(81.8 \%)$ & $14249(83.4 \%)$ & 3324 (91.1\%) \\
\hline Urban & 1470 (19.3\%) & $90(6.0 \%)$ & $1044(13.4 \%)$ & $173(9.5 \%)$ & 330 (19.6\%) & $60(18.2 \%)$ & $2844(16.6 \%)$ & $323(8.9 \%)$ \\
\hline \multicolumn{9}{|l|}{ Ethnicity } \\
\hline Kikongo & 0 & 0 & $6257(80.3 \%)$ & $1488(81.9 \%)$ & $1680(100 \%)$ & 330 (100\%) & 7937 (46.4\%) & 1818 (49.9\%) \\
\hline Kimbundo & 0 & 0 & $1536(19.7 \%)$ & $328(18.1 \%)$ & 0 & 0 & $1536(9.0 \%)$ & 328 (9.0\%) \\
\hline Umbundo & $7620(100 \%)$ & $1501(100 \%)$ & 0 & 0 & 0 & 0 & 7620 (44.6\%) & $1501(41.2 \%)$ \\
\hline \multicolumn{9}{|l|}{ Ecological zone } \\
\hline Northern-coastal & 0 & 0 & 0 & 0 & $930(55.4 \%)$ & $210(63.6 \%)$ & $930(5.4 \%)$ & $210(5.8 \%)$ \\
\hline Coffee & 0 & 0 & $2553(32.8 \%)$ & 738 (40.6\%) & $750(44.6 \%)$ & $120(36.4 \%)$ & 3303 (19.3\%) & $858(23.5 \%)$ \\
\hline Central highland & $7620(100 \%)$ & $1501(100 \%)$ & $5240(67.2 \%)$ & 1078 (59.4\%) & 0 & 0 & $12860(75.2 \%)$ & $2579(70.7 \%)$ \\
\hline
\end{tabular}

\section{Prevalence and infection intensity}

When considering RDT trace readings as positive, the overall prevalence (adjusted for clustering) of $S$. mansoni was $21.2 \%$ (95\%Cl 18.1, 24.6) and S. haematobium was $13.6 \%(95 \% \mathrm{Cl} 11.9,15.6)$, with an overall prevalence of any schistosomiasis of $31.4 \%(95 \% \mathrm{Cl} 28.2,34.7)(\mathrm{Table} 2)$. When considering RDT trace readings as negative, the overall prevalence (adjusted for clustering) of $S$. mansoni was $11.8 \%(95 \% \mathrm{Cl} 9.7,14.3)$ and $S$. haematobium was $8.4 \%$ $(95 \% \mathrm{Cl} 7.1,9.9)$, with an overall prevalence of any schistosomiasis of $19.0 \%(95 \% \mathrm{Cl} 16.7,21.5)($ Table 2$)$. Additional File 2 provides the prevalence (adjusted for clustering) of schistosomiasis as determined by RDTs for each municipality. Figures 1, 2 and 3 show the spatial distribution of schistosomiasis infection for each municipality (as determined when considering RDT trace readings as positive) across Huambo, Uige and Zaire provinces accordingly. Of the 3,131 POC-CCA $\AA$ results with a detectable reading 1,406 (44.9\%) were trace readings, 1,052 (33.6\%) were "+", $400(12.8 \%)$ were "++", and $273(8.7 \%)$ were "+++". Of the 2,005 Hemastix $\circledast$ results with a detectable reading, 767 (38.3\%) were trace readings, $567(28.3 \%)$ were " + ", $299(14.9 \%)$ were "++", and $372(18.6 \%)$ were "+++". 
Table 2

Prevalence of schistosomiasis based on rapid diagnostic tests and microscopy for Huambo, Uige and Zaire provinces.

\begin{tabular}{|c|c|c|c|c|}
\hline & Huambo & Uige & Zaire & Total \\
\hline \multicolumn{5}{|l|}{ S. mansoni } \\
\hline RDT & $N=7620$ & $N=7793$ & $N=1680$ & $N=17093$ \\
\hline Overall when trace pos, \% (range) & $20.8(6.6-47.2)$ & $21.2(2.0-74.8)$ & $23.1(0.9-47.1)$ & $21.2(0.9-74.8)$ \\
\hline Overall when trace neg, \% (range) & $8.9(2.7-18.4)$ & $16.1(0.9-55.0)$ & $14.1(0-29.9)$ & $11.8(0-55.0)$ \\
\hline Microscopy & $N=1501$ & $N=1818$ & $N=330$ & $N=3649$ \\
\hline Light intensity, \% (95\%Cl) & $0.2(0.05,0.9)$ & $20.0(9.0,38.6)$ & $0.1(0.01,1.1)$ & $8.9(3.5,20.4)$ \\
\hline Moderate intensity, \% (95\%Cl) & 0 & $0.04(0.01,0.3)$ & 0 & $0.02(0.003,0.1)$ \\
\hline Heavy intensity, \% (95\%Cl) & 0 & 0 & 0 & 0 \\
\hline Overall, \% (range) & $0.2(0-0.8)$ & $20.0(0-87.4)$ & $0.1(0-1.3)$ & $8.9(0-87.4)$ \\
\hline \multicolumn{5}{|l|}{ S. haematobium } \\
\hline RDT & $N=7620$ & $N=7793$ & $N=1680$ & $N=17093$ \\
\hline Overall when trace pos, \% (range) & $18.7(0-29.8)$ & $5.6(0.7-9.9)$ & $11.3(3.8-31.2)$ & $13.6(0-31.2)$ \\
\hline Overall when trace neg, \% (range) & $11.9(0-20.8)$ & $2.8(0.3-9.6)$ & $7.0(0-18.0)$ & $8.4(0-20.8)$ \\
\hline Microscopy & $N=1500$ & $N=1615$ & $N=330$ & $N=3445$ \\
\hline Light intensity, \% (95\%Cl) & $5.7(3.3,9.6)$ & $0.6(0.2,1.7)$ & $1.7(0.3,8.7)$ & $3.4(2.1,5.4)$ \\
\hline Heavy intensity, \% (95\%Cl) & $2.7(1.3,5.5)$ & $0.1(0.05,0.4)$ & $1.6(0.2,12.7)$ & $1.6(0.8,3.1)$ \\
\hline Overall, \% (range) & $8.4(0-34.4)$ & $0.7(0-5.9)$ & $3.3(0-6.6)$ & $5.0(0-34.4)$ \\
\hline \multicolumn{5}{|l|}{ Any schistosomiasis } \\
\hline RDT & $N=7620$ & $N=7793$ & $N=1680$ & $N=17093$ \\
\hline Overall when trace pos, \% (range) & $34.7(26.9-57.0)$ & $25.3(5.9-77.3)$ & $32.2(6.9-51.2)$ & $31.4(5.9-77.3)$ \\
\hline Overall when trace neg, \% (range) & $19.1(12.5-31.5)$ & $18.4(3.5-55.6)$ & $20.1(1.6-34.1)$ & $19.0(1.6-55.6)$ \\
\hline Microscopy & $N=1501$ & $N=1818$ & $N=330$ & $N=3649$ \\
\hline Overall, \% (range) & $8.6(0-34.4)$ & $24.7(0-87.4)$ & $3.3(0-6.6)$ & $14.5(0-87.4)$ \\
\hline
\end{tabular}

For the subset of schoolchildren who submitted a sample for microscopy the overall prevalence (adjusted for clustering) of S. mansoni was 8.9\% (95\%Cl 3.6, $20.5)$ and S. haematobium was $5.0 \%(95 \% \mathrm{Cl} 3.1,8.0)$, with an overall prevalence of any schistosomiasis of $14.5 \%(95 \% \mathrm{Cl} 8.2,24.4)(\mathrm{Additional} \mathrm{File} 2)$. Nearly all S. mansoni infections were of light intensity (183/184, 98.5\%), while for S. haematobium 145/233 (62.2\%) were light and $88 / 233(37.8 \%)$ were heavy intensity infections. The overall prevalence (adjusted for clustering) of moderate or heavy intensity $\mathrm{S}$. mansoni infection was $0.2 \%(95 \% \mathrm{Cl} 0.003,0.1)$, and the overall prevalence (adjusted for clustering) of heavy intensity S. haematobium infection was $1.6 \%(95 \% \mathrm{Cl} 0.8,3.1)(\mathrm{Table} 2)$.

The overall prevalence (adjusted for clustering) of any STH infection across the three provinces was $29.5 \%(95 \% \mathrm{Cl} 23.2,36.6)(\mathrm{Table} 3)$. Ascaris Iumbricoides was the most common STH species, with an overall prevalence of $25.1 \%(95 \% \mathrm{Cl} 19.4,31.9)$ (Table 3$)$. The overall prevalence of hookworm was $5.2 \%(95 \% \mathrm{Cl} 3.7,7.3)$ and the overall prevalence of $T$. trichiura was $3.6 \%(95 \% \mathrm{Cl} 2.6,5.0)(\mathrm{Table} 3)$. Additional File 3 shows the prevalence of STH species for each municipality. Figures 1, 2 and 3 display the spatial representation of STH prevalence for each municipality across Huambo, Uige and Zaire provinces respectively. Nearly all STH infections were of light intensity, with 324/324 (100\%) hookworm, 1,114/1,126 (98.9\%) A. lumbricoides, and 167/170 (98.2\%) T. trichiura infections determined light intensity. The overall prevalence (adjusted for clustering) of moderate or heavy intensity infections was $0.7 \%$ $(95 \% \mathrm{Cl} 0.2,2.4)$ for $A$. lumbricoides, $0.04 \%(95 \% \mathrm{Cl} 0.01,0.2)$ for $T$. trichiura and $0 \%$ for hookworm (Table 3$)$. 
Table 3

Prevalence of soil-transmitted helminths by microscopy for Huambo, Uige and Zaire provinces.

\begin{tabular}{|c|c|c|c|c|}
\hline & Huambo & Uige & Zaire & Total \\
\hline Hookworm & $N=1501$ & $N=1816$ & $N=330$ & $N=3647$ \\
\hline Light intensity, \% $(95 \% \mathrm{Cl})$ & $0.1(0.02,1.1)$ & $11.1(7.2,16.8)$ & $4.2(0.9,18.1)$ & $5.2(3.7,7.3)$ \\
\hline Moderate intensity, \% $(95 \% \mathrm{Cl})$ & 0 & 0 & 0 & 0 \\
\hline Heavy intensity, \% $(95 \% \mathrm{Cl})$ & 0 & 0 & 0 & 0 \\
\hline Overall, \% (range) & $0.1(0-0.6)$ & $11.1(0-42.6)$ & $4.2(0-8.0)$ & $5.2(0-42.6)$ \\
\hline A. lumbricoides & $N=1501$ & $N=1816$ & $N=330$ & $N=3647$ \\
\hline Light intensity, \% (95\%Cl) & $12.4(6.6,21.9)$ & $39.4(30.1,49.6)$ & $16.5(8.7,28.9)$ & $24.5(19.1,30.8)$ \\
\hline Moderate intensity, $\%(95 \% \mathrm{Cl})$ & $0.1(0.02,0.9)$ & $1.4(0.4,5.0)$ & 0 & $0.7(0.2,2.4)$ \\
\hline Heavy intensity, \% (95\%Cl) & 0 & 0 & 0 & 0 \\
\hline Overall, \% (range) & $12.5(0-32.8)$ & $40.8(3.4-89.7)$ & $16.5(0-30.9)$ & $25.1(0-89.7)$ \\
\hline T. trichiura & $N=1496$ & $N=1813$ & $N=330$ & $N=3639$ \\
\hline Light intensity, \% (95\%Cl) & $0.6(0.2,1.4)$ & $7.1(5.2,9.7)$ & $2.4(0.8,6.7)$ & $3.6(2.5,5.0)$ \\
\hline Moderate intensity, \% $(95 \% \mathrm{Cl})$ & $0.08(0.02,0.4)$ & 0 & 0 & $0.04(0.01,0.2)$ \\
\hline Heavy intensity, \% (95\%Cl) & 0 & 0 & 0 & 0 \\
\hline Overall, \% (95\%Cl) & $0.7(0-4.2)$ & $7.1(0-24.2)$ & $2.4(0-7.4)$ & $3.6(0-7.4)$ \\
\hline Any STH & $N=1501$ & $N=1818$ & $N=330$ & $N=3647$ \\
\hline Overall, \% (range) & $13.1(0.8-33.2)$ & $49.4(5.2-89.7)$ & $20.6(6.7-36.8)$ & $29.5(0.8-89.7)$ \\
\hline
\end{tabular}

\section{Diagnostic performance of RDTs compared to microscopy}

Table 4 summarises the comparative diagnostic performance between schistosomiasis RDTs and microscopy. When considering RDT trace readings as positive, the diagnostic agreement between POC-CCA ${ }^{\circledR}$ and Kato-Katz in detecting $S$. mansoni infection was fair $(K=0.38$, $p<0.001)$, and the diagnostic agreement between Hemastix ${ }^{B}$ and urine filtration was moderate $(K=0.51, p<0.001)$. The specificity of the RDTs compared to microscopy was $88.8 \%$ $(95 \% \mathrm{Cl} 87.7,89.9)$ for POC-CCA $\AA$ and $92.7 \%(95 \% \mathrm{Cl} 91.7,93.5)$ for Hemastix $\circledast$. When considering RDT trace readings as negative, the diagnostic agreement between $\mathrm{POC}-\mathrm{CCA} \AA$ and Kato-Katz was moderate $(\mathrm{K}=0.57, \mathrm{p}<0.001)$, and the diagnostic agreement between Hemastix $\AA$ and urine filtration was moderate $(K=0.53, p<0.001)$. The specificity of the RDTs compared to microscopy was $95.2 \%(95 \% \mathrm{Cl} 94.4,95.9)$ for POC-CCA $\AA$ and $94.9 \%(95 \% \mathrm{Cl} 94.1,95.6)$ for Hemastix ${ }^{\circledR}$.

Table 4

Diagnostic performance between schistosomiasis rapid diagnostic tests and microscopy.

\begin{tabular}{|c|c|c|c|c|c|c|c|c|c|c|c|}
\hline & \multirow[t]{2}{*}{ Kat-Katz } & \multicolumn{5}{|c|}{ RDT (trace positive) } & \multicolumn{5}{|c|}{ RDT (trace negative) } \\
\hline & & Detected & $\begin{array}{l}\text { Not } \\
\text { detected }\end{array}$ & Agreement & $\begin{array}{l}\text { Kappa } \\
\text { statistic }^{a}\end{array}$ & $\begin{array}{l}\mathrm{p}- \\
\text { value }\end{array}$ & Detected & $\begin{array}{l}\text { Not } \\
\text { detected }\end{array}$ & Agreement & $\begin{array}{l}\text { Kappa } \\
\text { statistic }^{a}\end{array}$ & $\begin{array}{l}\mathrm{p}- \\
\text { value }\end{array}$ \\
\hline \multirow[t]{2}{*}{ S. mansoni } & Detected & 155 & 29 & $88.6 \%$ & 0.38 & $<0.001$ & 150 & 34 & $94.5 \%$ & 0.57 & $<0.001$ \\
\hline & $\begin{array}{l}\text { Not } \\
\text { detected }\end{array}$ & 387 & 3078 & & & & 167 & 3298 & & & \\
\hline \multirow{2}{*}{$\begin{array}{l}\text { S. } \\
\text { haematobium }\end{array}$} & Detected & 181 & 52 & $91.6 \%$ & 0.51 & $<0.001$ & 157 & 76 & $93.0 \%$ & 0.53 & $<0.001$ \\
\hline & $\begin{array}{l}\text { Not } \\
\text { detected }\end{array}$ & 236 & 2976 & & & & 164 & 3048 & & & \\
\hline
\end{tabular}

There were 225 samples that had a trace POC-CCA $®$ reading and were analysed by Kato-Katz for S. mansoni. Of these, $220 / 225$ ( $97.8 \%$ ) were negative on microscopy and 5/225 (2.2\%) were determined light intensity S. mansoni infection, with no moderate or heavy intensity $S$. mansoni infections identified. For S. haematobium, there were 96 samples that had a trace Hemastix ${ }^{\circledR}$ reading and were analysed by urine filtration. Of these $72 / 96(75.0 \%)$ were negative on microscopy, 18/96 (18.8\%) were determined light intensity infection and 6/96 (6.2\%) were determined heavy intensity infection. 


\section{School questionnaire on school WASH indicators}

Table 5 summarises the results of the school WASH questionnaire for each province. Of the 575 schools participating in the parasitological surveys there were 217 (37.7\%) schools that had functional bathrooms and $134(23.3 \%)$ that had a reliable safe drinking water source. There were $472 / 576(81.9 \%)$ that received school-based anthelminthic preventive chemotherapy in 2013. Additional File 4 displays results from the WASH questionnaire for all municipalities across Huambo, Uige and Zaire provinces.

Table 5

Results from school water, sanitation and hygiene questionnaires for Huambo, Uige and Zaire provinces.

\begin{tabular}{|lllll|}
\hline & Huambo & Uige & Zaire & Total \\
& $\mathbf{N}=\mathbf{2 5 4}$ & $\mathbf{N = 2 6 5}$ & $\mathbf{N}=\mathbf{5 6}$ & $\mathbf{N}=\mathbf{5 7 5}$ \\
\hline How many schools had bathrooms? & $\mathbf{n}(\%)$ & $\mathbf{n}(\%)$ & $\mathbf{n}(\%)$ & $\mathbf{n}(\%)$ \\
\hline How many schools had bathrooms in good condition? & $148(58.3)$ & $127(47.9)$ & $40(71.4)$ & $315(54.8)$ \\
\hline How many schools had a reliable safe drinking water source? & $86(33.9)$ & $40(15.1)$ & $8(14.3)$ & $134(23.3)$ \\
\hline Water source type? & & & & \\
\hline Tap & $63(24.8)$ & $20(7.5)$ & $2(3.6)$ & $85(14.8)$ \\
\hline Hole/others & $18(7.1)$ & $20(7.5)$ & $6(10.7)$ & $49(8.5)$ \\
\hline How many schools were dewormed in 2013? & $203(79.9)$ & $218(82.3)$ & $51(91.1)$ & $472(82.1)$ \\
\hline School Directors' demonstrated knowledge of schistosomiasis. & $81(31.9)$ & $55(20.8)$ & $29(51.8)$ & $165(28.7)$ \\
\hline N: number of schools participating. & & & & $217(37.7)$ \\
\hline
\end{tabular}

\section{Factors associated with schistosomiasis or STH infection}

On multivariate regression analysis, factors associated with S. mansoni included: ecological zone, with schoolchildren in the Central highland (aOR 2.90 $(95 \% \mathrm{Cl} 1.20,7.02), \mathrm{p}=0.02)$ or Coffee (aOR $23.21(95 \% \mathrm{Cl} 9.13,58.98), \mathrm{p}<0.01)$ ecological zones more likely to be infected compared to schoolchildren in the Northern-coastal ecological zone; and ethnicity, with schoolchildren of Kikongo (aOR $0.34(95 \% \mathrm{Cl} 0.24,0.49)$, p<0.001) or Kimbundo (aOR 0.40 (95\% Cl 0.21, $0.77), p=006$ ) ethnicity less likely to be infected than schoolchildren of Umbundo ethnicity (Table 6). For $S$. haematobium, schoolchildren in the Coffee (aOR $0.42(95 \% \mathrm{Cl} 0.19,0.91), \mathrm{p}=0.03)$ or Central highland (aOR $0.29(95 \% \mathrm{Cl} 0.13,0.64), \mathrm{p}=0.002)$ ecological zones were less likely to be infected compared to schoolchildren in the Northern-coastal ecological zone; as well as schoolchildren of Kikongo $(\mathrm{aOR} 0.21(95 \% \mathrm{Cl} 0.15,0.29)$, p<0.001) or Kumbundo ethnicity (aOR $0.36(95 \% \mathrm{Cl} 0.27,0.49), \mathrm{p}<0.001)$ were less likely to be infected than schoolchildren of Umbundo ethnicity. Other factors associated with $S$. haematobium infection included a higher odds for females (aOR $1.51(95 \% \mathrm{Cl} 1.27,1.80), \mathrm{p}<0.001)$ compared to males, age more than 12 years compared to age less than 11 years (aOR $1.76(95 \% \mathrm{Cl} 1.40,2.22), \mathrm{p}<0.001)$, and attending school in an urban setting $(\mathrm{aOR} 1.60(95 \% \mathrm{Cl} 1.17,2.18), \mathrm{p}<0.001) \mathrm{compared}$ to a rural setting (Table 6). Factors associated with any schistosomiasis infection are displayed in Table 6 . Additional File 5 shows the results of the multivariate regression analyses when considering schistosomiasis RDT trace readings as negative, which demonstrates similar associations between age, setting, ecological zone and ethnicity variables and any schistosomiasis. 
Table 6

Factors on multivariate analysis associated with schistosomiasis.

\begin{tabular}{|c|c|c|c|c|c|c|c|c|c|}
\hline & \multicolumn{3}{|l|}{ S. mansoni } & \multicolumn{3}{|c|}{ S. haematobium } & \multicolumn{3}{|c|}{ Any schistosomiasis } \\
\hline & $n / N$ & aOR [95\% Cl] & $p$ & $n / N$ & aOR [95\% Cl] & $p$ & $\mathrm{n} / \mathrm{N}$ & $\begin{array}{l}\text { aOR } \\
{[95 \% \mathrm{Cl}]}\end{array}$ & $p$ \\
\hline \multicolumn{10}{|l|}{ Sex } \\
\hline Male & $1574 / 8550$ & NS & NS & $855 / 8550$ & 1 & - & $2215 / 8550$ & 1 & - \\
\hline Female & $1557 / 8543$ & NS & NS & $1150 / 8543$ & $\begin{array}{l}1.51[1.27 \\
1.80]\end{array}$ & $<0.001$ & $2409 / 8543$ & $\begin{array}{l}1.16[1.02, \\
1.33]^{2}\end{array}$ & 0.02 \\
\hline \multicolumn{10}{|l|}{ Age group (years) } \\
\hline$<11$ & $1151 / 6348$ & NS & NS & $608 / 6348$ & 1 & & $1589 / 6348$ & 1 & - \\
\hline $11-12$ & $959 / 5436$ & NS & NS & $665 / 5436$ & $\begin{array}{l}1.31[1.00 \\
1.72]\end{array}$ & 0.05 & $1479 / 5436$ & $\begin{array}{l}1.09[0.92 \\
1.30]\end{array}$ & 0.31 \\
\hline$>12$ & $971 / 5204$ & NS & NS & $722 / 5204$ & $\begin{array}{l}1.76[1.40, \\
2.22]\end{array}$ & $<0.001$ & $1502 / 5204$ & $\begin{array}{l}1.24[1.00, \\
1.53]^{2}\end{array}$ & 0.046 \\
\hline \multicolumn{10}{|l|}{ School setting } \\
\hline Rural & $2508 / 14249$ & NS & NS & $1552 / 14249$ & 1 & - & $3655 / 14249$ & 1 & - \\
\hline Urban & $623 / 2844$ & NS & NS & $453 /, 844$ & $\begin{array}{l}1.60[1.17 \\
2.18]\end{array}$ & 0.03 & $969 / 2844$ & $\begin{array}{l}1.40[1.02 \\
1.91]\end{array}$ & 0.04 \\
\hline \multicolumn{10}{|l|}{$\begin{array}{l}\text { Ecological zone of } \\
\text { school }\end{array}$} \\
\hline Northern-coastal & $25 / 930$ & 1 & - & $133 / 930$ & 1 & - & $152 / 930$ & 1 & - \\
\hline Coffee & $1019 / 3303$ & $\begin{array}{l}23.21[9,13, \\
58.98]\end{array}$ & $<0.001$ & $228 / 3303$ & $\begin{array}{l}0.42[0.19 \\
0.91]\end{array}$ & 0.03 & $1165 / 3303$ & $\begin{array}{l}3.69[1.72 \\
7.89]\end{array}$ & 0.001 \\
\hline Central highland & $2087 / 12860$ & $2.90[1.20,7.02]$ & 0.02 & $1644 / 12860$ & $\begin{array}{l}0.29[0.13 \\
0.64]\end{array}$ & 0.002 & $3307 / 12860$ & $\begin{array}{l}0.70[0.35 \\
1.42]\end{array}$ & 0.32 \\
\hline \multicolumn{10}{|l|}{ Ethnicity } \\
\hline Umbundo & $1762 / 7620$ & 1 & - & $1413 / 7620$ & 1 & - & $2774 / 7620$ & 1 & - \\
\hline Kikongo & $1239 / 7937$ & $0.34[0.24,0.49]$ & $<0.001$ & 493/7937 & $\begin{array}{l}0.21[0.15 \\
0.29]\end{array}$ & $<0.001$ & $1637 / 7937$ & $\begin{array}{l}0.26[0.20 \\
0.35]\end{array}$ & $<0.001$ \\
\hline Kimbundo & $130 / 1536$ & $0.40[0.21,0.77]$ & 0.006 & $99 / 1536$ & $\begin{array}{l}0.36[0.27, \\
0.49]\end{array}$ & $<0.001$ & $213 / 1536$ & $\begin{array}{l}0.35[0.35, \\
0.53]\end{array}$ & $<0.001$ \\
\hline \multicolumn{10}{|l|}{$\begin{array}{l}\text { School dewormed } \\
2013\end{array}$} \\
\hline No & $575 / 3061$ & NS & NS & $355 / 3061$ & NS & NS & $853 / 3061$ & NS & NS \\
\hline Yes & $2556 / 14032$ & NS & NS & $1650 / 14032$ & NS & NS & $3771 / 14032$ & NS & NS \\
\hline \multicolumn{10}{|l|}{ Latrines at school } \\
\hline None/non-functional & $1816 / 10603$ & NS & NS & $1256 / 10603$ & NS & NS & $2759 / 10603$ & NS & NS \\
\hline Functional & $1315 / 6490$ & NS & NS & $749 / 6490$ & NS & NS & $1865 / 6490$ & NS & NS \\
\hline \multicolumn{10}{|l|}{ Water at school } \\
\hline Not available & $2269 / 13063$ & NS & NS & $1455 / 13063$ & NS & NS & $3345 / 13063$ & NS & NS \\
\hline Available & $862 / 4030$ & NS & NS & $550 / 4030$ & NS & NS & $1279 / 4030$ & NS & NS \\
\hline
\end{tabular}

Table 7 reports the factors on multivariate regression analysis associated with STH infection. For hookworm, there was a higher odds of infection for schoolchildren of Kikongo (aOR 68.83 (95\%Cl 8.44, 482.56), p<0.01) or Kumbundo (aOR 50.41 (95\% Cl 4.47, 568.21), p=0.002) ethnicity compared to schoolchildren of Kikongo ethnicity; while there was a lower odds of infection for schoolchildren at schools with functional latrines (aOR 0.35 ( $95 \mathrm{Cl} 0.03$, 0.14), $\mathrm{p}=0.02$ ) compared to those at schools without functional latrines. For $A$. lumbricoides there was a higher odds of infection for schoolchildren of Kikongo (aOR $6.91(95 \% \mathrm{Cl} 2.86,16.70), \mathrm{p}<0.001)$ or Kumbundo (aOR $9.21(95 \% \mathrm{Cl} 3.87,21.96), \mathrm{p}<0.001)$ ethnicity compared to Umbundo ethnicity, as well as for schoolchildren in the Central highland ecological zone (aOR $5.65(95 \% \mathrm{Cl} 1.79,17.82), \mathrm{p}=0.03)$ compared to those in the Northern-coastal ecological zone. For T. trichiura, there was a higher odds of infection for schoolchildren of Kikongo (aOR 10.35 (95\% Cl 4.25, 25.33), p<0.001) or Kumbundo (aOR 9.95 (95\%Cl 
$3.30,30.01), p<0.001)$ ethnicity compared to schoolchildren of Umbundo ethnicity, while females (aOR $0.40(95 \% \mathrm{Cl} 0.23,0.69)$, $\mathrm{p}=0.01)$ were found to be less likely to be infected than males. Overall, the only factor associated with any STH infection was ethnicity, with those from Kikongo (aOR 4.96 ( $95 \% \mathrm{Cl} 2.26$,

10.89), $\mathrm{p}<0.001)$ or Kumbundo (aOR $10.74(95 \% \mathrm{Cl} 4.95,23.31), \mathrm{p}<0.001)$ more likely to have a STH infection than schoolchildren from Umbundo ethnicity. 
Table 7

Factors on multivariate analysis associated with soil-transmitted helminth infection.

\begin{tabular}{|c|c|c|c|c|c|c|c|c|c|c|c|c|}
\hline & \multicolumn{3}{|l|}{ Hookworm } & \multicolumn{3}{|c|}{ A. Iumbricoides } & \multicolumn{3}{|l|}{ T. trichiura } & \multicolumn{3}{|l|}{ Any STH } \\
\hline & $n / N$ & $\begin{array}{l}\text { aOR } \\
{[95 \% \mathrm{Cl}]}\end{array}$ & $p$ & $n / N$ & $\begin{array}{l}\text { aOR } \\
{[95 \% \mathrm{Cl}]}\end{array}$ & $\mathbf{p}$ & $n / N$ & $\begin{array}{l}\text { aOR } \\
{[95 \% \mathrm{Cl}]}\end{array}$ & $p$ & $\mathrm{n} / \mathrm{N}$ & $\begin{array}{l}\text { aOR } \\
{[95 \% \mathrm{Cl}]}\end{array}$ & $p$ \\
\hline \multicolumn{13}{|l|}{ Sex } \\
\hline Male & $186 / 1818$ & 1 & - & $572 / 1818$ & NS & NS & $99 / 1813$ & 1 & - & $688 / 1818$ & 1 & - \\
\hline Female & $138 / 1829$ & $\begin{array}{l}0.72 \\
{[0.49} \\
1.07]\end{array}$ & 0.11 & $554 / 1829$ & NS & NS & $71 / 1826$ & $\begin{array}{l}0.40 \\
{[0.23} \\
0.69]\end{array}$ & 0.001 & $641 / 1829$ & $\begin{array}{l}0.82 \\
{[0.62} \\
1.08]\end{array}$ & 0.15 \\
\hline \multicolumn{13}{|l|}{$\begin{array}{l}\text { Age group } \\
\text { (years) }\end{array}$} \\
\hline$<11$ & $143 / 1394$ & 1 & - & $500 / 1394$ & 1 & - & $68 / 1391$ & 1 & - & $576 / 1394$ & 1 & - \\
\hline $11-12$ & $80 / 1096$ & $\begin{array}{l}0.74 \\
{[0.50} \\
1.09]\end{array}$ & 0.12 & $315 / 1096$ & $\begin{array}{l}0.84 \\
{[0.63} \\
1.12]\end{array}$ & 0.23 & $50 / 1092$ & $\begin{array}{l}1.10 \\
{[0.61} \\
1.98]\end{array}$ & 0.31 & $363 / 1096$ & $\begin{array}{l}0.79 \\
{[0.56} \\
1.11]\end{array}$ & 0.17 \\
\hline$>12$ & $101 / 1090$ & $\begin{array}{l}0.82 \\
{[0.54} \\
1.23]\end{array}$ & 0.33 & $310 / 1090$ & $\begin{array}{l}0.73 \\
{[0.44} \\
1.21]\end{array}$ & 0.21 & $52 / 1089$ & $\begin{array}{l}0.62 \\
{[0.32} \\
1.19]\end{array}$ & 0.15 & $389 / 1090$ & $\begin{array}{l}0.72 \\
{[0.43} \\
1.21]\end{array}$ & 0.22 \\
\hline \multicolumn{13}{|l|}{$\begin{array}{l}\text { School } \\
\text { setting }\end{array}$} \\
\hline Rural & $305 / 3324$ & NS & NS & $1028 / 3324$ & NS & NS & $158 / 3316$ & NS & NS & $1218 / 3324$ & NS & NS \\
\hline Urban & $19 / 323$ & NS & NS & $98 / 323$ & NS & NS & $12 / 323$ & NS & NS & $111 / 323$ & NS & NS \\
\hline \multicolumn{13}{|l|}{$\begin{array}{l}\text { Ecological } \\
\text { zone of } \\
\text { school }\end{array}$} \\
\hline $\begin{array}{l}\text { Northern- } \\
\text { coastal }\end{array}$ & $16 / 210$ & NS & NS & $41 / 210$ & 1 & - & $10 / 210$ & NS & NS & $58 / 210$ & NS & NS \\
\hline Coffee & $790 / 858$ & NS & NS & $285 / 858$ & $\begin{array}{l}2.49 \\
{[0.75} \\
8.25]\end{array}$ & 0.14 & $48 / 856$ & NS & NS & $335 / 858$ & NS & NS \\
\hline $\begin{array}{l}\text { Central } \\
\text { highland }\end{array}$ & $240 / 2579$ & NS & NS & $800 / 2579$ & $\begin{array}{l}5.65 \\
{[1.79} \\
17.82]\end{array}$ & 0.003 & $112 / 2573$ & NS & NS & $936 / 2579$ & NS & NS \\
\hline \multicolumn{13}{|l|}{ Ethnicity } \\
\hline Kikongo & $252 / 1818$ & 1 & - & $754 / 1818$ & 1 & - & $1672 / 1815$ & 1 & - & $910 / 1818$ & 1 & - \\
\hline Kimbundo & $70 / 328$ & $\begin{array}{l}0.79 \\
{[0.18} \\
3.38]\end{array}$ & 0.75 & $199 / 328$ & $\begin{array}{l}1.33 \\
{[0.61} \\
2.91]\end{array}$ & 0.47 & $12 / 328$ & $\begin{array}{l}0.96 \\
{[0.42} \\
2.19]\end{array}$ & 0.92 & $233 / 328$ & $\begin{array}{l}2.17 \\
{[1.19} \\
3.94]\end{array}$ & 0.01 \\
\hline Umbundo & $2 / 1501$ & $\begin{array}{l}0.02 \\
{[0.002} \\
0.12]\end{array}$ & $<0.001$ & $173 / 1501$ & $\begin{array}{l}0.14 \\
{[0.06} \\
0.35]\end{array}$ & $<0.001$ & $15 / 1496$ & $\begin{array}{l}0.10 \\
{[0.04} \\
0.24]\end{array}$ & $<0.001$ & $186 / 1501$ & $\begin{array}{l}0.20 \\
{[0.09} \\
0.44]\end{array}$ & $<0.001$ \\
\hline \multicolumn{13}{|l|}{$\begin{array}{l}\text { School } \\
\text { dewormed } \\
2013 / 2014\end{array}$} \\
\hline No & $86 / 714$ & 1 & - & $187 / 714$ & NS & NS & $33 / 713$ & NS & NS & $225 / 714$ & NS & NS \\
\hline Yes & $238 / 2933$ & $\begin{array}{l}0.71 \\
{[0.21} \\
2.38]\end{array}$ & 0.57 & $939 / 2933$ & NS & NS & $137 / 2926$ & NS & NS & $1104 / 2933$ & NS & NS \\
\hline \multicolumn{13}{|l|}{$\begin{array}{l}\text { Latrines at } \\
\text { school }\end{array}$} \\
\hline $\begin{array}{l}\text { None/non- } \\
\text { functional }\end{array}$ & $306 / 2462$ & 1 & - & $874 / 2462$ & NS & NS & $137 / 2,458$ & NS & NS & $1052 / 2462$ & NS & NS \\
\hline Functional & $18 / 1185$ & $\begin{array}{l}0.35 \\
{[0.03} \\
0.14]\end{array}$ & 0.03 & $252 / 1185$ & NS & NS & $33 / 1181$ & NS & NS & $277 / 1185$ & NS & NS \\
\hline
\end{tabular}




\begin{tabular}{|c|c|c|c|c|c|c|c|c|c|c|c|c|}
\hline & \multicolumn{3}{|l|}{ Hookworm } & \multicolumn{3}{|c|}{ A. lumbricoides } & \multicolumn{3}{|l|}{ T. trichiura } & \multicolumn{3}{|l|}{ Any STH } \\
\hline \multicolumn{13}{|l|}{$\begin{array}{l}\text { Safe } \\
\text { drinking } \\
\text { water at } \\
\text { school }\end{array}$} \\
\hline $\begin{array}{l}\text { Not } \\
\text { available }\end{array}$ & $314 / 2793$ & 1 & - & $987 / 2793$ & 1 & - & $147 / 2789$ & 1 & - & $1174 / 2793$ & 1 & - \\
\hline Available & $10 / 854$ & $\begin{array}{l}0.55 \\
{[0.11,} \\
2.83]\end{array}$ & 0.47 & $139 / 854$ & $\begin{array}{l}0.57 \\
{[0.22} \\
1.46]\end{array}$ & 0.24 & $23 / 850$ & $\begin{array}{l}0.58 \\
{[0.20} \\
1.69]\end{array}$ & 0.31 & $155 / 854$ & $\begin{array}{l}0.50 \\
{[0.19} \\
1.30]\end{array}$ & 0.15 \\
\hline
\end{tabular}

\section{Discussion}

This survey, which was carried out in 2014, represents one of the earlier experiences in using schistosomiasis RDTs as a component of a large-scale schoolbased prevalence survey, following the experience in Namibia,[7] and provides the risk-stratified rationale for the school-based preventive chemotherapy program with praziquantel and albendazole. As expected, there were differences in schistosomiasis prevalence based on whether the schistosomiasis RDT trace readings were considered positive or negative. However, this had little impact on risk stratification according to WHO-endorsed prevalence criteria, with the majority of municipalities found to be moderate to high risk schistosomiasis regions (prevalence $\geq 10 \%$ ) [1] irrespective of the interpretation of trace RDT readings. As for STH infection, there was variable STH distribution across municipalities, however only 2 of 11 municipalities in Huambo and 3 of 6 municipalities in Zaire had a STH prevalence $\geq 20 \%$. This may in part be due to the impact of prior deworming undertaken in schools throughout 2013 . However, it is uncertain as to whether the WHO preventive chemotherapy coverage goals of at least $75 \%$ of the target population [1] were achieved. Of particular concern is the prevalence of STH infections in Uige, with 15 of 16 municipalities with a STH prevalence $\geq 20 \%$.

How to interpret trace RDT in determining schistosomiasis infection across different settings has not been definitively established [15]. Of particular interest is the impact of the enhanced capacity for field work using RDTs that facilitate surveys from larger and more geographically representative populations. There was a small improvement in specificity for the RDTs when considering trace readings as negative compared to positive, as well as improved diagnostic agreement between POC-CCA ${ }^{\circledR}$ and Kato-Katz when RDT trace readings were considered negative, however there was no difference in diagnostic agreement between Hemastix ${ }^{\circledR}$ and urine filtration based on whether trace readings were considered positive or negative. Given the comparatively low detection of schistosomiasis on microscopy compared to RDTs, there is concern for the low sensitivity of microscopy methods in our survey, which hinders the ability to make more definitive conclusions as to whether RDT trace readings reflect true infection or not. The low sensitivity of microscopy methods in field surveys is well recognized, particularly in low-prevalence and light-intensity settings, which has prompted an increasing reliance on RDTs to estimate schistosomiasis prevalence $[8,11,12,14,16-18]$. This is in part due to the fluctuation in S. mansoni egg excretion and variable distribution of eggs in stool [19], with the variability in S. mansoni antigen excretion as detected by POC-CCA® shown to be less pronounced than egg excretion in stool for detection by Kato-Katz [20-22]. The lack of an established gold standard rapid diagnostic test for schistosomiasis and the increasingly recognized low sensitivity of Kato-Katz limits the capacity to accurately determine the prevalence of schistosomiasis and STH in the field [18, 23, 24]. Operational research is ongoing to evaluate and validate field diagnostics for schistosomiasis and STH infection, which will become increasingly important as countries strive toward elimination.

Our analysis identified both ecological zone and ethnicity to be associated with $S$. mansoni infection. Given that Umbundo ethnicity was exclusive to Huambo province in this survey, it is likely that the association with ethnicity is in part explained by other environmental, sociodemographic or behavioural differences between the provinces. As such it is difficult to ascertain as to whether any cultural practices specific to schoolchildren of Umbundo ethnicity place them at higher risk for $S$. mansoni infection compared to schoolchildren of Kikongo or Kimbundo ethnicity. For $S$. haematobium, females, older age, and urban school settings were found to be risk factors in addition to ecological zone and ethnicity characteristics. Previous studies demonstrate mixed results with respect to the association of age and sex with $S$. haematobium [25-30], which likely reflects local conventions relating to the home environment that would increase exposure to water sources, such as playing, fetching water or washing.

The main factor identified on our analysis to be associated with STH infection was ethnicity, with schoolchildren of Kikongo or Kimbundo ethnicity more likely to be infected with any of the STH species, as well overall STH infection, compared to schoolchildren of Umbundo ethnicity. As previously discussed, the association singling out Umbundo ethnicity as a lower risk group is likely to in part reflect other environmental, sociodemographic or behavioural differences between Huambo province and the others.

There are several limitations to be acknowledged with this survey. Firstly, only a single Kato-Katz smear was conducted on stool specimens to assess for $S$. mansoni and STH species, which is not in keeping with the WHO recommendations for double Kato-Katz smears and is likely to underestimate $S$. mansoni and STH prevalence in the microscopy survey [31]. This was done to facilitate time- and cost-efficient field work given the large distances field teams had to travel throughout the provinces. This limitation is further compounded by the operator-dependent nature of microscopy, which is reliant upon training of field workers with variable experience in specimen handling and microscopy. This survey is vulnerable to selection bias associated with only selecting schoolchildren present on the day of specimen collection. This may result in selecting from a population who are well enough to attend school, which risks underestimating the prevalence of schistosomiasis and STH infection. Lastly, there was limited data collected relating to school-based water, sanitation and hygiene measures, and no data relating to home or community activities and environment for schoolchildren that could be incorporated into the risk factor 
analysis. However, through the inclusion of ecological zone and ethnicity variables, some broader representation of the out-of-school context for these schoolchildren is represented.

\section{Conclusions}

This survey identified most municipalities across Huambo, Uige and Zaire met the prevalence threshold for a school-based schistosomiasis preventive chemotherapy program. As for STH, while the prevalence in many municipalities in Huambo and Zaire did not meet the WHO threshold for a school-based preventive chemotherapy program, there were a high proportion of schools that had received prior deworming. Despite this inability to discern an accurate baseline prevalence, this survey provided critical data for informing the next steps for a school-based STH preventive chemotherapy program. Ecological zone and ethnicity were factors associated with schistosomiasis and STH infection, which identifies the need for further evaluation of environmental, sociodemographic and behavioural factors at school and home to inform targeted control strategies to compliment preventive chemotherapy programs.

\section{Abbreviations}

\begin{tabular}{ll} 
aOR & \multicolumn{1}{l}{ Adjusted odds ratio } \\
CI & Confidence interval \\
DALY & Disease-adjusted life year \\
IQR & Inter-quartile range \\
NTD & Neglected tropical disease \\
POC-CCA & Point-of-care circulating cathodic antigen \\
RDT & Rapid diagnostic test \\
STH & Soil-transmitted helminth \\
WASH & Water, sanitation and hygiene \\
WHO & World Health Organization
\end{tabular}

\section{Declarations}

Ethics approval and consent to participate

Approval for the survey protocol was obtained by the Ministry of Public Health of Angola. Informed written consent was obtained from the school directors of each school to permit participation of children in the school-based surveys.

\section{Consent for publication}

Not applicable.

Availability of data and materials

The datasets used and/or analysed during the current study are available from the corresponding author on reasonable request.

Competing interests

The authors declare no competing interests.

Funding

This study was funded by The END Fund. Additional resources were provided by the Ministry of Health of Angola, the Provincial Health Directorates in Angola, and the National Institute of Statistics of Angola.

\section{Author's contributions}

AWB analysed and interpreted the data, and drafted the manuscript. JCF designed and implemented the study, conducted initial data analysis, and reviewed the manuscript. RCvG managed and analysed the data, and reviewed the manuscript. PM reviewed the study protocol, trained and supervised field teams, coordinated field logistics, oversaw quality control of data collection, and reviewed the manuscript. WL reviewed the study protocol, oversaw the conduct of the field work, and reviewed the manuscript. RM trained and supervised field teams, coordinated field logistics, and reviewed manuscript. EM supervised implementation of field work, interpreted the data, and reviewed the manuscript. SVN supervised data analysis, interpretation of data and drafting of manuscript. SL trained and supervised field teams, coordinated field logistics, and reviewed manuscript. All authors read and approved and the final manuscript. 
The authors would like to acknowledge all the children, families, field workers, school directors, teachers, the MENTOR Initiative technicians, and the Ministry of Education of Angola and its provincial delegates that participated and facilitated the study. The authors would also like to acknowledge the Ministry of Health of Angola, particularly Dr Pedro Van Dunem, Director of the Neglected Tropical Diseases Program. We would like to thank the National Institute of Statistics of Angola and the Provincial Health Directorates in Angola for allowing use of their vehicles and fuel in Huambo and Uige respectively.

Author's information

Not applicable.

\section{References}

1. World Healt Organization. Helminth control in school-age children: A guide for managers of control programmes.

https://apps.who.int/iris/bitstream/handle/10665/44671/9789241548267_eng.pdf?sequence=1\&isAllowed=y (2011). Accesssed 14 Oct 2021.

2. Hay SI, Abajobir AA, Abate KH, Abbafati C, Abbas KM, Abd-Allah F, et al. Global, regional, and national disability-adjusted life-years (DALYs) for 333 diseases and injuries and healthy life expectancy (HALE) for 195 countries and territories, 1990-2016: a systematic analysis for the Global Burden of Disease Study 2016. Lancet. 2017;390(10100):1260-344.

3. World Health Organization. Ending the neglect to attain the sustainable development goals: A road map for neglected tropical diseases $2021-2030$. https://www.who.int/publications/i/item/9789240010352 (2020). Accessed 14 October 2021.

4. World Health Organization. Schistosomiasis: progress report 2001 - 2011, strategic plan 2012 - 2020. https://apps. who.int/iris/handle/10665/78074 (2013). Accessed 14 October 2021.

5. World Health Organization. Accelerating work to overcome the global impact of neglected tropical diseases: a roadmap for implementation : executive summary. https://apps.who.int/iris/bitstream/handle/10665/70809/WHO_HTM_NTD_2012.1_eng.pdf?sequence=1\&isAllowed=y (2012). Accessed 14 October 2021.

6. World Health Organization. 2030 targets for soil-transmitted helminthiases control programmes.

https://apps.who.int/iris/bitstream/handle/10665/330611/9789240000315-eng.pdf?sequence=1\&isAllowed=y (2020). Accessed 14 October 2021.

7. Sousa-Figueiredo JC, Stanton MC, Katokele S, Arinaitwe M, Adriko M, Balfour L, et al. Mapping of Schistosomiasis and Soil-Transmitted Helminths in Namibia: The First Large-Scale Protocol to Formally Include Rapid Diagnostic Tests. PLoS Negl Trop Dis. 2015;9(7):e0003831.

8. Clark NJ, Umulisa I, Ruberanziza E, Owada K, Colley DG, Ortu G, et al. Mapping Schistosoma mansoni endemicity in Rwanda: a critical assessment of geographical disparities arising from circulating cathodic antigen versus Kato-Katz diagnostics. PLoS Negl Trop Dis. 2019;13(9):e0007723.

9. Knopp S, Ame SM, Hattendorf J, Ali SM, Khamis IS, Bakar F, et al. Urogenital schistosomiasis elimination in Zanzibar: accuracy of urine filtration and haematuria reagent strips for diagnosing light intensity Schistosoma haematobium infections. Parasit Vectors. 2018;11(1):552.

10. Leta GT, Mekete K, Wuletaw Y, Gebretsadik A, Sime H, Mekasha S, et al. National mapping of soil-transmitted helminth and schistosome infections in Ethiopia. Parasit Vectors. 2020;13(1):437.

11. Ortu G, Ndayishimiye O, Clements M, Kayugi D, Campbell CH, Lamine MS, et al. Countrywide Reassessment of Schistosoma mansoni Infection in Burundi Using a Urine-Circulating Cathodic Antigen Rapid Test: Informing the National Control Program. Am J Trop Med Hyg. 2017;96(3):664-73.

12. Ruberanziza E, Wittmann U, Mbituyumuremyi A, Mutabazi A, Campbell CH, Colley DG, et al. Nationwide Remapping of Schistosoma mansoni Infection in Rwanda Using Circulating Cathodic Antigen Rapid Test: Taking Steps toward Elimination. Am J Trop Med Hyg. 2020;103(1):315-24.

13. Sousa-Figueiredo JC, Gamboa D, Pedro JM, Fancony C, Langa AJ, Magalhaes RJ, et al. Epidemiology of malaria, schistosomiasis, geohelminths, anemia and malnutrition in the context of a demographic surveillance system in northern Angola. PloS One. 2012;7(4):e33189.

14. Bocanegra C, Gallego S, Mendioroz J, Moreno M, Sulleiro E, Salvador F, et al. Epidemiology of Schistosomiasis and Usefulness of Indirect Diagnostic Tests in School-Age Children in Cubal, Central Angola. PLoS Negl Trop Dis. 2015;9(10):e0004055.

15. Clements MN, Donnelly CA, Fenwick A, Kabatereine NB, Knowles SCL, Meite A, et al. Interpreting ambiguous 'trace' results in Schistosoma mansoni CCA Tests: Estimating sensitivity and specificity of ambiguous results with no gold standard. PLoS Negl Trop Dis. 2017;11(12):e0006102.

16. Kittur N, Castleman JD, Campbell CH, King CH, Colley DG. Comparison of Schistosoma mansoni Prevalence and Intensity of Infection, as Determined by the Circulating Cathodic Antigen Urine Assay or by the Kato-Katz Fecal Assay: A Systematic Review. Am J Trop Med Hyg. 2016;94(3):605-10.

17. Adriko M, Standley CJ, Tinkitina B, Tukahebwa EM, Fenwick A, Fleming FM, et al. Evaluation of circulating cathodic antigen (CCA) urine-cassette assay as a survey tool for Schistosoma mansoni in different transmission settings within Bugiri District, Uganda. Acta Trop. 2014;136:50-7.

18. Clements MN, Corstjens P, Binder S, Campbell CH Jr, de Dood CJ, Fenwick A, et al. Latent class analysis to evaluate performance of point-of-care CCA for low-intensity Schistosoma mansoni infections in Burundi. Parasit Vectors. 2018;11(1):111.

19. Krauth SJ, Coulibaly JT, Knopp S, Traore M, N'Goran EK, Utzinger J. An in-depth analysis of a piece of shit: distribution of Schistosoma mansoni and hookworm eggs in human stool. PLoS Negl Trop Dis. 2012;6(12):e1969.

20. Van Etten L, Engels D, Krijger FW, Nkulikyinka L, Gryseels B, Deelder AM. Fluctuation of schistosome circulating antigen levels in urine of individuals with Schistosoma mansoni infection in Burundi. Am J Trop Med Hyg. 1996;54(4):348-51.

21. Polman K, Engels D, Fathers L, Deelder AM, Gryseels B. Day-to-day fluctuation of schistosome circulating antigen levels in serum and urine of humans infected with Schistosoma mansoni in Burundi. Am J Trop Med Hyg. 1998;59(1):150-4. 
22. Mwinzi PN, Kittur N, Ochola E, Cooper PJ, Campbell CH Jr, King CH, et al. Additional Evaluation of the Point-of-Contact Circulating Cathodic Antigen Assay for Schistosoma mansoni Infection. Front Public Health. 2015;3:48.

23. Amoah AS, Hoekstra PT, Casacuberta-Partal M, Coffeng LE, Corstjens P, Greco B, et al. Sensitive diagnostic tools and targeted drug administration strategies are needed to eliminate schistosomiasis. The Lancet Infectious diseases. 2020;20(7):e165-e72.

24. Cavalcanti MG, Cunha AFA, Peralta JM. The Advances in Molecular and New Point-of-Care (POC) Diagnosis of Schistosomiasis Pre- and Postpraziquantel Use: In the Pursuit of More Reliable Approaches for Low Endemic and Non-endemic Areas. Front Immunol. 2019;10:858.

25. Augusto G, Nala R, Casmo V, Sabonete A, Mapaco L, Monteiro J. Geographic distribution and prevalence of schistosomiasis and soil-transmitted helminths among schoolchildren in Mozambique. Am J Trop Med Hyg. 2009;81(5):799-803.

26. Kabuyaya M, Chimbari MJ, Manyangadze T, Mukaratirwa S. Efficacy of praziquantel on Schistosoma haematobium and re-infection rates among school-going children in the Ndumo area of uMkhanyakude district, KwaZulu-Natal, South Africa. Infect Dis Poverty. 2017;6(1):83.

27. Geleta S, Alemu A, Getie S, Mekonnen Z, Erko B. Prevalence of urinary schistosomiasis and associated risk factors among Abobo Primary School children in Gambella Regional State, southwestern Ethiopia: a cross sectional study. Parasit Vectors. 2015;8:215.

28. Kapito-Tembo AP, Mwapasa V, Meshnick SR, Samanyika Y, Banda D, Bowie C, et al. Prevalence distribution and risk factors for Schistosoma hematobium infection among school children in Blantyre, Malawi. PLoS Negl Trop Dis. 2009;3(1):e361.

29. Degarege A, Mekonnen Z, Levecke B, Legesse M, Negash Y, Vercruysse J, et al. Prevalence of Schistosoma haematobium Infection among School-Age Children in Afar Area, Northeastern Ethiopia. PloS one. 2015;10(8):e0133142.

30. Ismail HA, Hong ST, Babiker AT, Hassan RM, Sulaiman MA, Jeong HG, et al. Prevalence, risk factors, and clinical manifestations of schistosomiasis among school children in the White Nile River basin, Sudan. Parasit Vectors. 2014;7:478.

31. Levecke B, Brooker SJ, Knopp S, Steinmann P, Sousa-Figueiredo JC, Stothard JR, et al. Effect of sampling and diagnostic effort on the assessment of schistosomiasis and soil-transmitted helminthiasis and drug efficacy: a meta-analysis of six drug efficacy trials and one epidemiological survey. Parasitology. 2014;141(14):1826-40.

\section{Figures}

Schistosomiasis prevalence

Huambo province

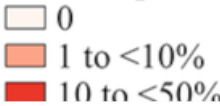

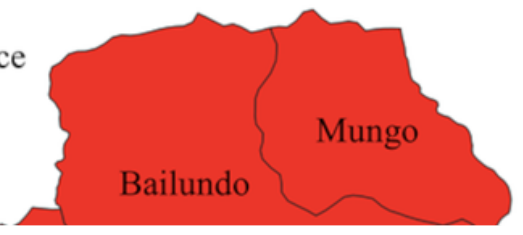

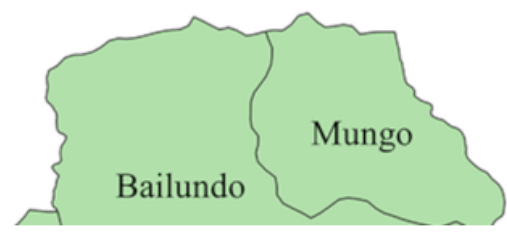

\section{Figure 1}

Prevalence of schistosomiasis and soil-transmitted helminth (STH) infections for Huambo province (based on rapid diagnostic tests, considering readings as positive). 

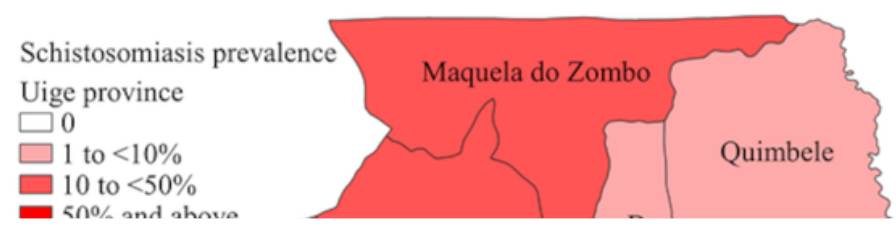

STH prevalence

Uige province

$\square 0$

$\square 1$ to $<20 \%$

20 to $<50 \%$

- $50 \%$ and above

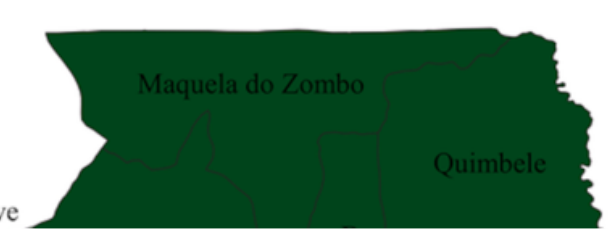

\section{Figure 2}

Prevalence of schistosomiasis and soil-transmitted helminth (STH) infections for Huambo province (based on rapid diagnostic tests, considering readings as positive).

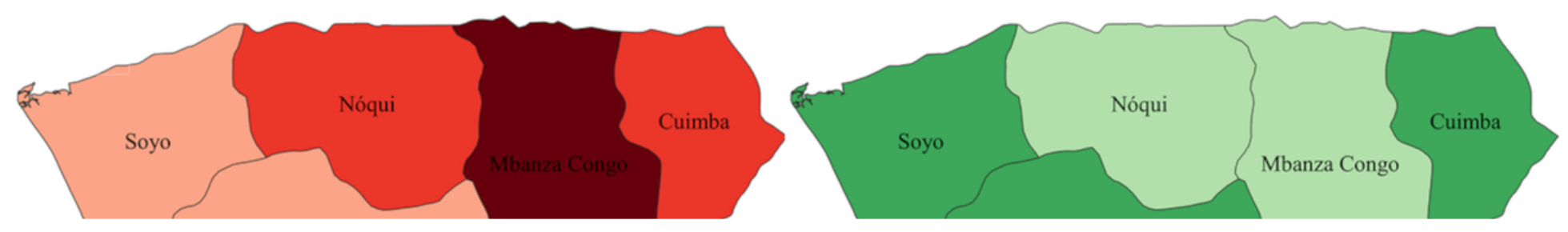

\section{Figure 3}

Prevalence of schistosomiasis and soil-transmitted helminth (STH) infections for Huambo province (based on rapid diagnostic tests, considering readings as positive).

\section{Supplementary Files}

This is a list of supplementary files associated with this preprint. Click to download.

- AdditionalFiles.docx 